\title{
PHONON INDUCED DRAG OF CHARGE CARRIERS IN HETEROSTRUCTURES IN MAGNETIC FIELDS: LIMIT OF WEAK FIELDS
}

\author{
Cz. Jasiukiewicz ${ }^{a}$, D. LemMANN ${ }^{b}$ and T. PASzkiewicz ${ }^{a}$ \\ ${ }^{a}$ Institute of Theoretical Physics, University of Wrocław \\ Pl. M. Borna 9, 50-204 Wrocław, Poland \\ ${ }^{b}$ Technische Universität Dresden, Institut für Theoretische Physik \\ Mommsenstr. 13, 01062 Dresden, Germany
}

\begin{abstract}
The response of a 2D gas of charge carriers of mobility $\mu$ in magnetic field $B$ to pulsed phonon beams is considered. Previously we derived a quantum Langevin equation for the centre-of-mass of the carrier gas, which allows us to calculate the time integrated drag current. This formula is studied in the weak magnetic field limit. When the ratio of the cyclotron frequency $\omega_{c}$ to the frequency $\omega$ of phonons is small and $\mu B \ll 1$, the general formula coincides with the corresponding expression obtained in the frame of the Boltzmann equation with the collision integral independent of $B$.
\end{abstract}

PACS numbers: $63.20 . \mathrm{Kr}, 73.40 .-\mathrm{c}$

\section{Introduction}

Let us consider a 2D gas of charge carriers lying in a plane perpendicular to a DC magnetic field $\boldsymbol{B}$ of the strength $B$. They are scattered by defects, the mean time elapsing between successive collisions is $\tau_{\mathrm{e}}$. The transport properties of this gas are characterized by the mobility $\mu=|e| \tau_{\mathrm{e}} / \mathrm{m}^{*}$, where $m^{*}$ and $e$ are respectively the effective mass and the charge of a carrier.

The field strength is characterized by comparing: (i) the cyclotron angular frequency $\omega_{\mathrm{c}}=e B / m^{*}$ to the inverse of the mean time $\tau_{\mathrm{e}}$ (or $\mu B$ to the unity); (ii) for $2 \mathrm{DG}$ of electrons $-\hbar \omega_{\mathrm{c}}$ to the spacing $\Delta E$ between the energy levels of carriers in a triangular well. Here we shall confine ourselves to the case of the fields which are not extremely strong, i.e. $\hbar \omega_{\mathrm{c}} \ll \Delta E$.

In magnetic field the charge transport is related to the translational motion of the centre-of-mass (CM) of 2DCG. Recently we have derived the quantum Langevin equation for the CM valid for $0<\mu B \ll \Delta E /\left(\hbar \tau_{\mathrm{e}}^{-1}\right)[1]$.

We also considered the non-stationary phonon drag effect and obtained the expression for the time integrated density of induced current (TICD for short). Besides being dependent on $\mu B$, TICD is also conditioned by the ratio of the 
frequency of absorbed phonon $\omega$ to $\omega_{c}$. The purpose of this note is to study the dependence of TICD [1] on $\omega_{c} / \omega$. In particular, we shall show that for $\omega>\omega_{c}$, i.e. when the transfer of energy is greater than the spacing between the adjacent Landau levels, the quasiclassical approximation is valid. If additionally $\mu B \ll 1$, the transport of charge is due to the translational motion of carriers. In such fields the motion of carriers can be treated semiclassically on the basis of the Boltzmann equation with the collision integral calculated for $B=0$. Such situation was considered in our previous paper [2].

\section{Time integrated drag current induced by pulsed phonon beams in 2D gases of carriers in magnetic fields}

We denote the deviation of the phonon distribution function from the Planck function by $\delta N(Q, r, t)$, where $Q$ stands for a pair $(q, j), q$ is the phonon wave vector, $j$ enumerates the three polarizations and $\omega(Q)$ is the angular frequency of the phonon $Q$. The component of $q$ lying in the gas plane is $q_{\perp}$, the component parallel to $B$ is $\boldsymbol{q}_{\|}$.

The carrier-phonon interaction is characterized by the matrix element $h(Q)$ containing contribution of the deformation potential and the piezoelectric coupling.

Using a procedure of Hu and O'Connel [3] we obtained the quantum Langevin equation for the CM of 2DCG [1]. We shall neglect the Coulomb interaction of carriers, many phonons absorption processes and mixed phonon-impurity processes. After taking the average over all impurities we obtain for the TICD in RPA

$$
\int_{-\infty}^{\infty} \mathrm{d} t j(t) \equiv \bar{j}(0)=\frac{\tau_{\mathrm{c}}}{\sqrt{1+(\mu B)^{2}}}\left[\boldsymbol{F}_{\mathrm{ph}}^{\mathrm{abs}}(0)+\mu B \boldsymbol{F}_{\mathrm{ph}}^{\mathrm{abs}}(0) \times \widehat{\boldsymbol{B}}\right],
$$

where $\vec{j}(\Omega)$ is the Fourier time transform of the current density, $\omega$ is the phonon frequency and

$$
\boldsymbol{F}_{\mathrm{ph}}^{\mathrm{abs}}(\Omega)=\frac{\pi}{V} \sum_{\boldsymbol{q}, j} \boldsymbol{q}_{\perp} \mathcal{N}(\Omega)|h(Q)|^{2} \sum_{m, m^{\prime}} \mathcal{L}_{m, m^{\prime}}\left(\omega, q_{\perp}, B\right)\left|G_{m, m^{\prime}}\left(q_{\|}\right)\right|^{2},
$$

where the function $\mathcal{L}_{m, m^{\prime}}$ plays an important role in our discussion

$$
\begin{aligned}
& \mathcal{L}_{m, m^{\prime}}\left(\omega, q_{\perp}, B\right) \\
& \quad=\sum_{n, n^{\prime}} C_{n, n^{\prime}}\left(q_{\perp} l_{B}\right)\left[f\left(\varepsilon_{n^{\prime}, m^{\prime}}\right)-f\left(\varepsilon_{n, m l}\right)\right] \delta\left[\varepsilon_{n^{\prime}, m^{\prime}}-\varepsilon_{n, m}-\hbar \omega(Q)\right] .
\end{aligned}
$$

Above $\mathcal{N}(\Omega)$ is the Fourier transform of the phonon deviation function, $l_{B}=$ $\sqrt{\hbar / e B}$ is the magnetic length and the function $C_{n, n^{\prime}}$ is related to the associated Laguerre polynomials $L_{n}^{\left(n-n^{\prime}\right)}(y)$

$$
C_{n n^{\prime}}(x)=\frac{1}{2 \pi l_{B}^{2}} \frac{n !}{n^{\prime} !} \frac{x^{2\left(n-n^{\prime}\right)}}{2^{\left(n-n^{\prime}\right)}} \exp \left(-x^{2} / 2\right)\left[L_{n}^{\left(n-n^{\prime}\right)}\left(x^{2} / 2\right)\right]^{2} .
$$

The form factor $\left|G_{m, m^{\prime}}\left(q_{\|}\right)\right|^{2}$ results from localization of carriers in the direction perpendicular to the surface of the (quasi-) 2DCG. The indices $m, m^{\prime}$ enumerate the subbands due to confinement perpendicular to the 2DCG plane and Landau levels due to magnetic quantization are labeled by the indices $n, n^{\prime}$. 
For weak magnetic fields $\left(\omega_{\mathrm{c}}<\omega\right)$ and for carriers occupying only the lowest subband ( $m=m^{\prime}=0$ ) the formula (1) should coincide with the quasiclassical expression for TICD [2]. The comparison of these formulae gives

$$
\lim _{B \rightarrow 0} \mathcal{L}_{0,0}\left(\omega, q_{\perp}, B\right)=-\frac{m^{*}}{2 \pi^{2} \hbar^{2} q_{\perp}} \mathcal{L}\left(\omega, q_{\perp}\right)
$$

The function $\mathcal{L}\left(\omega, q_{\perp}\right)$ was introduced previously [2]

$$
\mathcal{L}\left[\omega, q_{\perp}\right]=\operatorname{Re}\left(\sqrt{k_{\perp \mathrm{F}}^{2}-L_{-}^{2}}-\sqrt{k_{\perp \mathrm{F}}^{2}-L_{+}^{2}}\right),
$$

and $L_{ \pm}=q_{\perp} / 2 \pm m^{*} \omega /\left(\hbar q_{\perp}\right)$. the gas.

Here $k_{\perp \mathrm{F}}=\sqrt{2 \pi n_{\mathrm{s}}}$ is the Fermi wave vector and $n_{\mathrm{s}}$ is the surface density of

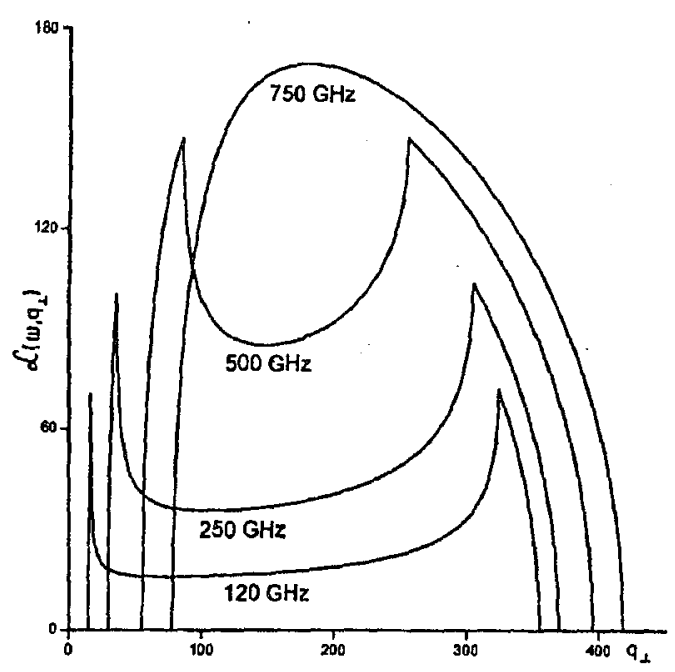

Fig. 1. Dependence of $\mathcal{L}\left(\omega, q_{\perp}\right)$ on $q_{\perp}$ for different values of $f=\omega / 2 \pi$. Axes units are $(\mu \mathrm{m})^{-1}$.

The calculations were performed for data corresponding to experiments on gas of holes in (001) plane of the GaAs/AlGaAs heterostructure [2]. The surface density of holes is $3300 \times 10^{12} \mathrm{~m}^{-2}, m^{*} \approx 0.4 m_{\mathrm{e}}$. In Fig. 1 we show the dependence of $\mathcal{L}\left(\omega, q_{\perp}\right)$ on $\omega$ and on $q_{\perp}$. In Figs. 2a-d we depict the dependence of $q_{\perp} \mathcal{L}_{0,0}\left(\omega, q_{\perp}, B\right)$ on $B$ for different values of $f=\omega / 2 \pi$. The oscillating lines with large amplitudes correspond to $\omega=\omega_{\mathrm{c}}$, while the oscillating lines with smaller amplitudes correspond to $\omega=5 \omega_{c}$. The non-oscillating curves represent the function $q_{\perp} \mathcal{L}\left(\omega, q_{\perp}\right)$. Figures $2 \mathrm{a}-\mathrm{d}$ show that when $\omega_{\mathrm{c}} / \omega$ diminishes, the amplitude of oscillations becomes smaller, so for $B \rightarrow 0\left(\omega_{c} / \omega \rightarrow 0\right)$ the function $\mathcal{L}_{0,0}\left(\omega, q_{\perp}, B\right)$ gradually goes to $\left(-m^{*} / 2 \pi^{2} \hbar^{2} q_{\perp}\right) \mathcal{L}\left(\omega, q_{\perp}\right)$. 

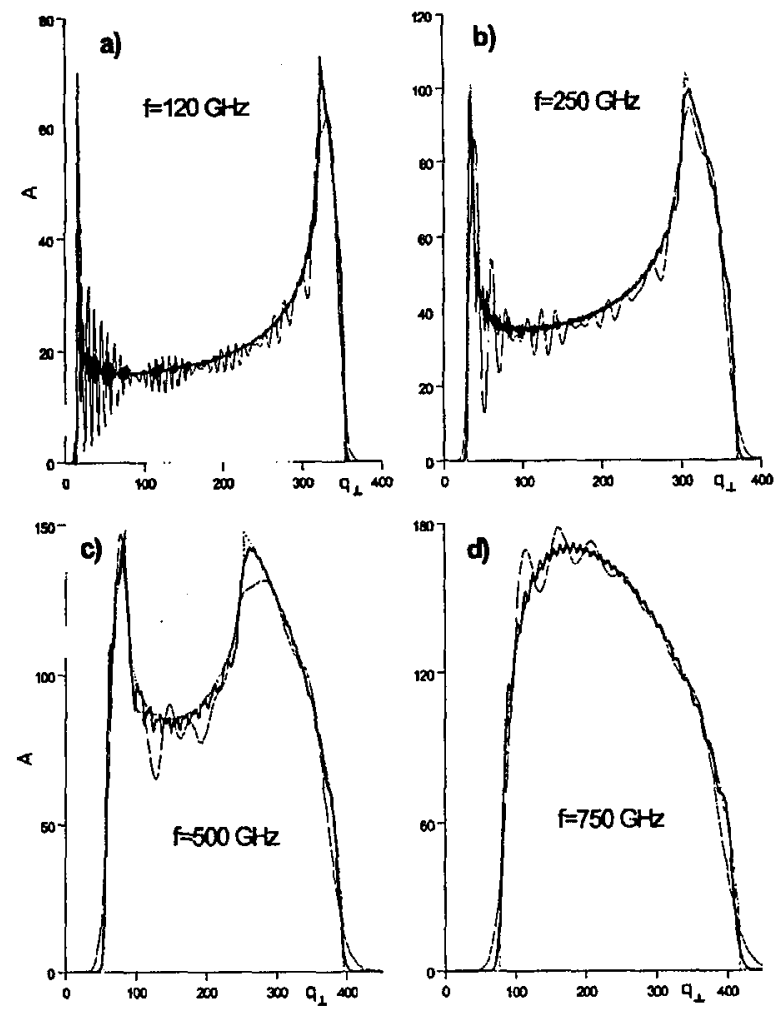

Fig. 2. Dependence of the function $A=\left(-m^{*} / 2 \pi^{2} \hbar^{2} q_{\perp}\right) \mathcal{L}_{0,0}\left(\omega, q_{\perp}, B\right)$ on $q_{\perp}$ for different values of $B$ and $f=\omega / 2 \pi$. Axes units are $(\mu \mathrm{m})^{-1}$.

This work was supported by EC Programme under the grant No. CIPACT 930070 and by the Committee for Scientific Research (Poland) under the grant No. PO $3 B 15708$.

\section{References}

[1] A.J. Kent, D. Lehmann, Cz. Jasiukiewicz, R.E. Strickland, K.R. Strickland, T. Paszkiewicz, in preparation.

[2] W.M. Gańcza, Cz. Jasiukiewicz, A.J. Kent, D. Lehmann, T. Paszkiewicz, K.R. Strickland, R.E. Strickland, Semicond. Sci. Technology, in press.

[3] G.Y. Hu, R.F. O'Connell, Physica A 151, 33 (1988). 